Philosophia

Scientie

\title{
Philosophia Scientiæ
}

Travaux d'histoire et de philosophie des sciences

18-1 | 2014

Standards of Rigor in Mathematical Practice

\section{La rigueur mathématique chez Henri Poincaré}

\section{Ramzi Kebaïli}

\section{OpenEdition}

\section{Journals}

Édition électronique

URL : http://journals.openedition.org/philosophiascientiae/910

DOI : 10.4000/philosophiascientiae.910

ISSN : 1775-4283

Éditeur

Éditions Kimé

Édition imprimée

Date de publication : 15 mars 2014

Pagination : 27-44

ISBN : 978-2-84174-665-1

ISSN : $1281-2463$

\section{Référence électronique}

Ramzi Kebailli, «La rigueur mathématique chez Henri Poincaré », Philosophia Scientiæ [En ligne], 18-1 |

2014, mis en ligne le 15 mars 2017, consulté le 02 novembre 2020. URL : http://

journals.openedition.org/philosophiascientiae/910 ; DOI : https://doi.org/10.4000/

philosophiascientiae.910 


\title{
La rigueur mathématique chez Henri Poincaré
}

\author{
Ramzi Kebaïli \\ Université Paris-Diderot, \\ UMR 7219 SPHERE (France)
}

Résumé : Henri Poincaré était réputé être un mathématicien hostile à la rigueur, aussi bien dans sa pratique mathématique que dans ses réflexions philosophiques. Or, des éléments indiquent que Poincaré se basait implicitement sur une conception personnelle de la rigueur mathématique, et qui correspondrait à sa pratique des mathématiques. Nous proposons donc de caractériser ce que serait cette conception. Tout d'abord, nous observons donc son rapport à la rigueur dans ses travaux en topologie, à partir d'exemples tirés du mémoire Analysis Situs de 1895 [Poincaré 1895]. Ensuite, nous étudions pour quelles raisons Poincaré revendique son opposition à certaines normes de rigueur. Enfin, nous développons une conception « poincaréenne » de la rigueur mathématique.

Abstract: Henri Poincaré had a reputation for being a mathematician hostile to rigor, as much in his mathematical practice as in his philosophical thoughts. But some elements show that Poincaré had implicitly in mind a personal conception of mathematical rigor, that would fit with his mathematical practice. We propose to develop what this conception would be. First, we observe his standards of rigor in his topological work, with some examples taken from his 1895 paper Analysis Situs [Poincaré 1895]. Then, we study on what grounds Poincaré claims his opposition to some specific standards of rigor. Finally, we develop a "Poincarean" conception of mathematical rigor.

\section{Introduction}

Henri Poincaré est l'un des plus grands mathématiciens de tous les temps. Mais il est également connu pour ses erreurs célèbres, par exemple, dans 
le mémoire Sur le problème des trois corps et les équations de la dynamique qui lui valut l'attribution du prix Oscar II en 1889 (à ce sujet, voir [Barrow-Greene 1997]).

En plus de ces cas caractérisés d'erreurs mathématiques, son style est réputé être assez négligé. Par exemple, dans son éloge posthume de Poincaré, Gaston Darboux écrit :

Mais, il ne faut pas craindre de le dire, si l'on veut donner une idée précise de la manière dont travaillait Poincaré; bien des points demandaient des corrections ou des explications. Poincaré était un intuitif. Une fois au sommet, il ne revenait jamais sur ses pas. Il se contentait d'avoir brisé les difficultés, et laissait aux autres le soin de tracer les routes royales qui devaient conduire plus facilement au but. [Darboux 1913, 15]

Comme nous le verrons, Poincaré a également été sévèrement critiqué par Jean Dieudonné dans A History of Algebraic and Differential Topology pour le manque de rigueur de ses mémoires topologiques [Dieudonné 1989].

Or, dans l'ensemble de ses écrits philosophiques, Poincaré insiste sur le fait que les mathématiques ne doivent pas se réduire à la rigueur logique, et que «l'intuition» y joue un rôle fondamental. Au premier abord, on pourrait se contenter de penser que Poincaré était principalement intéressé par la découverte de nouvelles idées mathématiques (qu'il attribuerait à l'intuition) et qu'il se souciait peu de leur mise en forme technique, ce qui serait somme toute assez banal pour un mathématicien de son envergure. Néanmoins, comme nous allons le voir, il a dans ses écrits philosophiques refusé de se cantonner à une opposition superficielle entre intuition heuristique et rigueur démonstrative, puisqu'il a soutenu que l'intuition était nécessaire à la démonstration mathématique.

En soutenant cette thèse, Poincaré s'opposait de manière virulente aux logiciens (notamment Russell et Whitehead) et reprochait à leur «logistique » d'entraver le travail du mathématicien ${ }^{1}$. En un certain sens, la rigueur — du moins telle que cette notion est comprise par les logiciens - serait donc stérilisante. Il y aurait donc dans le travail des mathématiciens une tension entre la rigueur et la fécondité. Mais en réalité, il s'agit là d'un faux dilemme : en effet, Poincaré refuse la vision logicienne de ce qui constitue l'essence même de la preuve mathématique, en dressant par exemple une comparaison avec

1. En 1906, il dit notamment : « Je ne vois au contraire dans la logistique que des entraves pour l'inventeur; elle ne nous fait pas gagner en concision, loin de là, et s'il faut 27 équations pour établir que 1 est un nombre, combien en faudra-t-il pour démontrer un vrai théorème. Si nous distinguons, avec $M$. Whitehead, l'individu $x$, la classe dont le seul membre est $x$ et qui s'appellera $\{x\}$, puis la classe dont le seul membre est la classe dont le seul membre est $x$ et qui s'appellera $\{\{x\}\}$, croit-on que ces distinctions, si utiles qu'elles soient, vont beaucoup alléger notre allure?» [Poincaré 1906, 295]. 
le jeu d'échecs en $1902^{2}$. Nous nous appuyons ici sur la thèse déjà développée par Michael Detlefsen dans [Detlefsen 1992], selon laquelle Poincaré aurait en tête une vision «alternative » de la rigueur. Selon Detlefsen, la vision «logicienne » de la démonstration mathématique, qui décompose celle-ci en une succession de déductions logiques, ne donne pas de compréhension d'ensemble de cette démonstration. Et c'est précisément ce manque de compréhension qui constituerait un manque de rigueur au sens «poincaréen ».

Or, comme nous allons le voir, les remarques de Poincaré sur la rigueur ne se limitent pas au problème (déjà crucial) de la compréhension globale. Notre objectif est donc de proposer une interprétation exhaustive des réflexions de Poincaré sur la rigueur, et qui découlerait de sa propre pratique des mathématiques. Fondamentalement, nous faisons donc l'hypothèse qu'il existe bien une telle conception unifiée qui serait sous-jacente aux remarques de Poincaré, et qui permettrait de répondre (au moins partiellement) aux critiques de Dieudonné. Mais encore faut-il préciser quels types de manquements à la rigueur sont visés par celui-ci. Pour évaluer les normes de rigueur chez Poincaré, nous allons commencer par étudier un exemple tiré de son mémoire Analysis Situs qui marque la création d'une nouvelle discipline, la topologie, dont les concepts de base n'étaient pas a priori clairement délimités. Nous allons ensuite présenter quelles sont les critiques portées par Poincaré contre deux types de normes de rigueur, celles des logiciens et celles des analystes, et enfin proposer une interprétation de ce que serait la rigueur «poincaréenne». Dans cette dernière partie, notre critère sera d'essayer de donner une interprétation unifiant différentes pistes suggérées par Poincaré, ainsi que sa pratique mathématique.

\section{L'absence de rigueur dans l'Analysis Situs}

Henri Poincaré lui-même considérait ses travaux en topologie comme étant parmi les plus fondamentaux de son œuvre. Ainsi, dans l'analyse qu'il livre de ses propres travaux en 1901, et qui ne sera publiée qu'en 1921, il insiste sur «l'importance extrême de cette science ${ }^{3} »$. De plus, en 1912, il ajoute :

2. «Si vous assistez à une partie d'échecs, il ne vous suffira pas, pour comprendre la partie, de savoir les règles de la marche des pièces. Cela vous permettrait seulement de reconnaître que chaque coup a été joué conformément à ces règles et cet avantage aurait vraiment bien peu de prix. C'est pourtant ce que ferait le lecteur d'un livre de mathématiques, s'il n'était que logicien. Comprendre la partie, c'est tout autre chose ; c'est savoir pourquoi le joueur avance telle pièce plutôt que telle autre qu'il aurait pu faire mouvoir sans violer les règles du jeu. C'est apercevoir la raison intime qui fait de cette série de coups successifs une sorte de tout organisé. À plus forte raison, cette faculté est-elle nécessaire au joueur lui-même, c'est-à-dire à l'inventeur »[Poincaré 1902, 125].

3. Il précise : «Quant à moi, toutes les voies diverses où je m'étais engagé successivement me conduisaient à l'Analysis Situs. J'avais besoin des données de cette science 
Et voici ce qui fait pour nous l'intérêt de cette Analysis Situs; c'est que c'est là qu'intervient vraiment l'intuition géométrique. [Poincaré 1912, 484]

Le mémoire Analysis Situs de 1895 [Poincaré 1895], ainsi que ses cinq compléments, sont à l'origine de la topologie en tant que discipline propre, et en particulier de notre topologie algébrique moderne. Nous assistons donc à la naissance d'une discipline.

En tant que discipline en cours de formation, et dont les concepts ne sont pas encore clairement dégagés, l'Analysis Situs se prête particulièrement à une étude de la méthodologie de recherche scientifique de Poincaré. Comme nous allons le voir, on note chez Poincaré différents types de manque de rigueur dans ce travail, comme le souligne Jean Dieudonné [Dieudonné 1989].

Ainsi, après avoir donné plusieurs définitions des variétés (sans préciser les liens entre elles), et plusieurs définitions des homologies, Poincaré va se retrouver à effectuer une démonstration complètement erronée du théorème de dualité. Mais nous allons expliquer pourquoi ce cas d'erreur mathématique illustre la méthodologie de Poincaré.

Rappelons tout d'abord l'objet du mémoire Analysis Situs. En termes modernes, il s'agit d'étudier certains sous-espaces de $\mathbb{R}^{n}$ en ne regardant que leurs propriétés restant inchangées par certaines déformations ${ }^{4}$, propriétés qui jouent un rôle capital dans plusieurs branches des mathématiques. Poincaré commence par définir les objets de son étude, appelés «variétés », puis il définit les déformations permises sur ces objets, appelées « homéomorphismes ». Riemann, puis Betti, avaient eu l'idée d'encoder les propriétés d'une variété à $k$ dimensions par la donnée de $k-1$ nombres $P_{1}, \ldots, P_{k-1}$ (les « nombres de Betti ») où — de manière heuristique - le nombre $P_{j}$ correspondrait au nombre de trous de dimension $j$ que possède la variété. L'espoir de départ était que cet encodage serait complet, dans le sens où deux variétés ayant les mêmes nombres de Betti seraient les mêmes du point de vue topologique (c'est-à-dire homéomorphes). Mais Poincaré va montrer que la donnée de ces nombres est insuffisante pour caractériser les variétés homéomorphes, et il propose pour y

pour poursuivre mes études sur les courbes définies par les équations différentielles et pour les étendre aux équations différentielles d'ordre supérieur et en particulier à celles du problème des trois corps. J'en avais besoin pour l'étude des fonctions non uniformes de 2 variables. J'en avais besoin pour l'étude des périodes des intégrales multiples et pour l'application de cette étude au développement de la fonction perturbatrice. Enfin, j'entrevoyais dans l'Analysis Situs un moyen d'aborder un problème important de la théorie des groupes, la recherche des groupes discrets ou des groupes finis contenus dans un groupe continu donné » [Poincaré 1921, 101].

4. En 1903 il précise : « Les théorèmes de l'Analysis Situs ont donc ceci de particulier qu'ils resteraient vrais si les figures étaient copiées par un dessinateur malhabile qui altérerait grossièrement toutes les proportions et remplacerait les droites par des lignes plus ou moins sinueuses. En termes mathématiques, ils ne sont pas altérés par une «transformation ponctuelle» quelconque » [Poincaré 1903, 285]. 
remédier d'associer également aux variétés un certain groupe $\mathrm{e}^{5}$. Un des objectifs du mémoire de Poincaré est de légitimer l'importance fondamentale de ce groupe en construisant deux variétés ayant les mêmes nombres de Betti mais des groupes associés différents (et donc non-homéomorphes). Mais pour parvenir à ce but, encore reste-t-il à définir correctement les nombres de Betti (ce qui nécessite de définir préalablement les « relations d'homologie », et d'être capable de les calculer. D'où l'importance cruciale d'obtenir une démonstration du Théorème de Dualité (pour une variété de dimension $n, P_{n-k}=P_{k}$ ), qui fournit un moyen de calculer facilement les nombres de Betti.

Pour évaluer les normes de rigueur à l'œuvre chez Poincaré, nous allons voir différents exemples précis d'absence de rigueur : dans sa définition des variétés, dans sa définition des homéomorphismes, dans sa définition des homologies, et enfin dans la détermination de ces homologies. Enfin, nous verrons comment un de ses manques de rigueur a conduit à une véritable erreur mathématique dans la démonstration du théorème de dualité, ce qui a nécessité l'écriture d'un Complément à l'Analysis Situs [Poincaré 1899].

Le mémoire commence par une «première définition des variétés » (c'est le titre de la première section $)^{6}$. Poincaré considère $x_{1}, x_{2}, \ldots, x_{n} n$ variables réelles regardées comme les coordonnées d'un point dans l'espace à $n$ dimensions. Il prend ensuite un système formé de $p$ égalités de la forme $F_{k}\left(x_{1}, \ldots, x_{n}\right)=0$ et $q$ inégalités de la forme $\phi_{j}\left(x_{1}, \ldots, x_{n}\right)>0$, en imposant comme premières conditions que les fonctions $F_{k}$ et $\phi_{j}$ soient uniformes ${ }^{7}$, continues et à dérivées continues. Il demande également que si on considère la matrice de taille $n \times p$ formée à la $k$-ème ligne et la $i$-ème colonne par les dérivées partielles $d F_{k} / d x_{i}$ évaluées en un point, ses sous-matrices de taille $p \times p$ ne soient jamais toutes de déterminant nul. Une fois tout ceci posé, Poincaré écrit :

Je dirais que l'ensemble des points qui satisfont aux conditions (1), s'il y en a, ce que je suppose, forme une variété à $n-p$ dimensions. [Poincaré 1895, 196]

L'objet ainsi défini est très proche de notre notion moderne de variété différentielle ${ }^{8}$.

5. Cette idée est considérée comme l'acte de naissance de la topologie algébrique, discipline qui associe à chaque objet topologique un objet algébrique, généralement un groupe. Cependant, cette appellation est anachronique car pour Poincaré, la théorie des groupes ne fait pas partie de l'algèbre. Il porte en effet une conception des groupes différente de la nôtre, point que nous ne détaillerons pas ici, puisque nous allons uniquement considérer des passages traitant des nombres de Betti.

6. Ce type de définition est parfois appelé « analytique», mais nous préférons éviter ici ce terme, car il reviendra dans l'article avec des sens différents.

7. C'est-à-dire que l'image d'un point est unique, condition que nous considérons aujourd'hui comme déjà intégrée dans la définition d'une fonction.

8. Pour toutes les traductions en termes modernes, nous nous référons à Jean Dieudonné, History of Algebraic and Differential Topology [Dieudonné 1989], sauf une exception qui sera signalée. 
Donnons un exemple pour bien comprendre ce que Poincaré a en tête. Par exemple, dans l'espace $\mathbb{R}^{3}$ formé des points à coordonnées réelles $\left(x_{1}, x_{2}, x_{3}\right)$, le système $\left\{1-x_{1}^{2}-x_{2}^{2}-x_{3}^{2}>0\right\}$ définit une variété à trois dimensions, qui est une boule ouverte $B$, et le système $\left\{1-x_{1}^{2}-x_{2}^{2}-x_{3}^{2}>0, x_{1}^{2}+x_{2}^{2}+x_{3}^{2}-1 / 2>0\right\}$ définit une boule creuse $K$, qui a également deux dimensions (puisqu'il n'y a que des inégalités). Autres exemples, le système $\left\{1-x_{1}^{2}-x_{2}^{2}-x_{3}^{2}=0\right\}$ définit une variété à deux dimensions, qui est la sphère $S_{1}$ de centre $(0,0,0)$ et de rayon 1 , et le système $\left\{x_{1}^{2}+x_{2}^{2}+x_{3}^{2}-1 / 2=0\right\}$ définit une autre sphère, que nous appellerons $S_{2}$.

Cette définition à partir d'un système d'équations et d'inéquations présente certains avantages, le premier d'entre eux étant de pouvoir déterminer la dimension d'une variété à partir du nombre d'égalités. De plus, cette approche permet à Poincaré de définir la notion de variété « continue » comme étant une variété où l'on peut passer de n'importe quel point à n'importe quel autre en les faisant «varier d'une manière continue » et «sans que les relations (1) cessent d'être satisfaites $»$. Poincaré ne précise pas ce qu'il entend par une variation faite « de manière continue », mais il n'y a pas de cercle vicieux contrairement à ce que les termes paraissent suggérer : la continuité d'une variété n'est pas de même nature que la continuité d'une application, et celle-ci peut se définir aisément si l'on admet l'analyse réelle déjà constituée. En termes modernes, Poincaré définit ce que nous appellerions aujourd'hui un espace connexe par $\operatorname{arcs}^{9}$. Dans la suite, il ne considérera que ce type de variétés.

Un autre avantage de ce type de définition, c'est de pouvoir définir la « frontière »d'une variété, ainsi : pour une variété $V$ de dimension $d$ donnée, sa frontière est constituée par toutes les variétés de dimension $d-1$ obtenues en remplaçant n'importe laquelle des inégalités qui définissent $V$ par une égalité. Dans nos exemples, la frontière de $B$ est formée par une seule variété, $S_{1}$, et la frontière de $K$ est constituée de $S_{1}$ et $S_{2}$. On notera au passage qu'ainsi définie, la frontière d'une variété n'est jamais incluse au sens ensembliste dans la variété, mais que Poincaré considère néanmoins que la frontière «fait partie » de la variété. Enfin, Poincaré appelle «fermée » une variété continue, bornée et dont la frontière est vide.

Cette définition des variétés est donc très commode pour définir des notions qui vont jouer un rôle capital par la suite. Cependant, à partir de la troisième section, il va construire de nouveaux objets qu'il va continuer de considérer comme des variétés, mais dans un sens différent. Il revendique cette démarche et le fait qu'il donne une « deuxième définition des variétés » (c'est le titre de la section). En réalité, c'est même deux nouvelles définitions qu'il donne : la première d'entre elles ne pose pas de problème, c'est une définition paramétrique par un système de $n$ équations $x_{i}=\theta\left(t_{1}, \ldots, t_{n}\right)$ et $m$ inégalités $\phi_{j}\left(t_{1}, \ldots, t_{n}\right)>0$, les fonctions étant prises supposées continues et même analytiques. Il n'y a pas de difficultés pour étendre les notions données pré-

9. Dieudonné traduit l'idée de Poincaré par espace «connexe», mais ceci nous semble incorrect. 
cédemment à cette nouvelle approche. Mais il ajoute ensuite une nouvelle définition, qu'il appelle le «procédé de la continuation analytique». Son idée est que si les frontières de deux variétés (continues) possèdent des éléments en commun, alors on peut considérer le recollage de ces deux variétés le long de cette frontière commune comme étant lui-même une nouvelle variété (continue), en un sens élargi. Il forme ainsi des « chaînes » de variétés, qui n'ont pas nécessairement de représentation paramétrique.

Poincaré aurait pu commencer par donner une seule définition générale de ce qu'est une variété, mais il ne l'a pas fait. Pourquoi? Une hypothèse serait que du point de vue pédagogique, il préférait avancer pas à pas et élargir progressivement sa notion pour que le lecteur comprenne bien la marche du raisonnement. Néanmoins, le plus probable, c'est que toutes les manipulations qu'il s'autorise sur les variétés définies de la première manière nécessiteraient de pénibles justifications pour être étendues en toute généralité.

Or, il y a un point vraiment problématique dans l'argumentation de Poincaré : c'est qu'il définit un critère d'équivalence topologique entre deux variétés distinctes au deuxième paragraphe, puis ne revient jamais dessus et n'explique pas comment celui-ci s'étendrait aux nouvelles définitions données dans la troisième section. Plus précisément, il définit une «équivalence du point de vue de l'Analysis Situs » qu'il appelle «homéomorphisme » (qui ne correspond pas exactement à notre notion actuelle d'homéomorphisme, mais plutôt à celle de difféomorphisme). Deux variétés sont homéomorphes au sens de Poincaré s'il existe entre elles une bijection dérivable, dont la dérivée est continue et inversible. Dans nos exemples, la boule $B$ est homéomorphe à toute autre boule, mais n'est pas homéomorphe à la sphère $S_{1}$, ni à $K$. La définition qu'a donnée Poincaré est plus restrictive que la définition moderne, qui demande simplement une bijection continue d'inverse continue. Mais quoi qu'il en soit, cette définition ne joue presque aucun rôle dans la suite de son mémoire. En revanche, il invoque souvent un autre critère d'équivalence, qui ne doit pas être confondu avec l'homéomorphie, qu'il désigne par les termes de variétés «peu différentes» ou encore «infiniment proches».

Or, ce critère non précisé joue un rôle capital dans la définition des nombres de Betti, comme nous allons le voir. Dans la cinquième section, Poincaré définit les relations d'homologie en dimension $m$ pour une variété $W$ à $d$ dimensions de la sorte : si $V_{1}, \ldots, V_{n}$ sont des variétés à $m$ dimensions formant la frontière d'une variété fixée à $m+1$ dimensions «faisant partie de $\mathrm{W} »$, on pose $V_{1}+\ldots+V_{n} \sim 0$. Par exemple dans la boule $B$, on a vu que la sphère $S_{1}$ formait sa frontière et donc on a $S_{1} \sim 0$, et dans $K$ on a $S_{1}+S_{2} \sim 0$. Ensuite à partir de ces équations posées, il stipule que «les homologies peuvent se combiner comme des équations ordinaires ». Concrètement dans la suite de son mémoire, il s'autorise les manipulations algébriques formelles suivantes :

- Si $V_{1}, V_{2}, \ldots, V_{n}$ sont $n$ variétés «peu différentes », on peut remplacer $V_{1}+V_{2}+\ldots+V_{n}$ par $n . V_{1}$ dans la relation d'homologie.

- Si $V_{1}+V_{2}+\ldots+V_{n} \sim 0$, on pose $V_{2}+\ldots+V_{n} \sim-V_{1}$. 
- Si $n . V_{1} \sim m . V_{2}$, où $m$ et $n$ entiers relatifs et $n$ non-nul, on pose $V_{1} \sim$ $(m / n) . V_{2}$. (Il est important de souligner pour la suite, que durant tout ce mémoire un flou persiste sur le caractère licite de cette opération de division).

Ce faisant, Poincaré commet une nouvelle entorse manifeste à la rigueur, qui est la suivante : il avait déjà défini dans la quatrième section ce que signifiait le symbole $-V$. Cette variété serait constituée des mêmes points que la variété $V$ (elles seraient donc identiques du point de vue ensembliste), mais avec une représentation différente. En effet, pour l'obtenir à partir d'une variété définie de la première manière, on permute l'ordre de deux équations du système, et pour une variété définie paramétriquement, on permute l'ordre de deux variables (par contre, il n'explique pas pour la troisième définition). Or, il n'a pas vérifié que cette définition du symbole $-V$ coïncidait avec celle donnée par le système des homologies (dans l'exemple que nous avions donné, $S_{1} \sim-S_{2}$ dans $K$, mais ceci ne pose pas de problème car $-S_{2}$ n'avait pas été défini auparavant).

Ceci posé, Poincaré définit dans la sixième section pour une variété $V$ à $n$ dimensions les nombres de Betti $P_{k}$, pour $k$ compris entre 1 et $n-1$. $P_{k}$ est défini comme étant le nombre minimal de variétés fermées à $k$ dimensions qui sont liées par une relation d'homologie dans $V$. Mais cette définition n'est pas adaptée pour des calculs effectifs : même dans des cas élémentaires, il serait trop fastidieux de la vérifier intégralement, et d'ailleurs Poincaré donne des exemples sans justifier. Il affirme ainsi que pour $B, P_{1}=1$ et $P_{2}=2$, et que pour $K, P_{1}=1$ et $P_{2}=1$, mais sans en donner de démonstration. Toutefois, ce sont à l'époque de Poincaré des exemples déjà connus, qui correspondent à l'idée intuitive que $P_{k}$ est le «nombre de trous à $k$ dimensions » augmenté d'une unité ${ }^{10}$.

Jusque-là, le manque de rigueur de Poincaré s'est caractérisé par des définitions flottantes et par des preuves évasives voire absentes. Ceci semblait toutefois ne pas toucher à la justesse de ses idées, et présenter l'avantage de montrer comment les notions se définissent par tâtonnements et élargissements progressifs. Pourtant, Poincaré va commettre une véritable erreur mathématique qui sera relevée par Poul Heegaard, comme il l'explique en préface de son Complément à l'Analysis Situs [Poincaré 1899]. En effet, dans la neuvième section, il prétend démontrer un théorème de dualité, à savoir que pour une variété fermée à $n$ dimensions, $P_{n-k}=P_{k}$. Or, Heegaard exhibe ce qui semble être un contre-exemple tout à fait acceptable selon les critères de Poincaré. Celui-ci reconnaît que sa démonstration était fausse, mais affirme que le contre-exemple de Heegaard n'en est un que si on interdit l'opération de division sur les homologies, ce qui donne lieu à deux notions distinctes de «nombres de Betti ». Ainsi, nous nous retrouvons avec deux notions qui semblent compatibles avec

10. Dans notre exemple, la boule creuse aurait un trou à deux dimensions, correspondant au prélèvement d'une boule intérieure, mais pas de trous à une dimension. 
la définition originelle de Poincaré, mais qui ne coïncident pas avec certaines variétés (comme celle exhibée par Heegaard). Or, ce qui est intéressant ici, c'est que Poincaré reconnaît que cette définition alternative ne correspond pas à la notion qu'il visait au départ, mais qu'il la maintient quand même comme étant légitime du fait qu'elle permet d'obtenir le théorème de dualité.

Notre interprétation est que confronté à un choix entre deux définitions distinctes, Poincaré a tout simplement choisi celle qui conduit à un théorème intéressant (car celui-ci fournit une symétrie dans les nombres de Betti), quand bien même celui-ci nécessite une distorsion de l'idée originelle. En ce sens, le manque de rigueur doit donc être pleinement assumé comme étant inscrit dans la pratique mathématique, puisque le flou dans les définitions employées permet justement de réajuster celles-ci en fonction de nouveaux objectifs qui peuvent apparaître en cours de route, comme celui de fournir un théorème important : en ce sens, ce seraient donc parfois les théorèmes qui justifieraient les définitions, et non l'inverse.

Ainsi, nous avons vu que l'Analysis Situs de Poincaré doit être lu plus comme une exposition de ses recherches avec une progression dans la généralité, que comme une exposition systématique. Or, cette démarche se justifie non seulement au point de vue pédagogique, mais également au niveau proprement mathématique : les concepts en question peuvent avoir plusieurs définitions possibles, et ce n'est qu'en cours de route qu'on trouvera des arguments nouveaux pour préférer l'une ou l'autre. Notre interprétation est donc qu'il y a dans la pratique de Poincaré une démarche volontaire de refus de certaines normes de rigueur, par exemple en évitant de donner des définitions précises, ou des preuves complètes, et que ceci se révèle être non pas une lubie mais une nécessité pour le développement des mathématiques. Mais pour que ce développement ne soit pas entravé, il est indispensable de distinguer soigneusement les cas de flou nécessaire et les cas d'erreurs mathématiques manifestes ; en somme il est indispensable de faire prévaloir une conception alternative de la rigueur.

\section{Le refus assumé de certaines normes de rigueur}

La question de la rigueur est souvent abordée chez Poincaré, notamment dans [Poincaré 1898], [Poincaré 1902], [Poincaré 1904] et [Poincaré 1905]. Bien qu'il adopte une attitude très critique, il est néanmoins soucieux de critiquer les manques de rigueur des mathématiciens du passé, comme en 1904 :

Longtemps les objets dont s'occupaient les mathématiciens étaient mal définis ; on croyait les connaître parce qu'on se les représentait avec les sens ou l'imagination, mais on en n'avait qu'une image 
grossière et non une idée précise sur laquelle le raisonnement pût avoir prise. [Poincaré 1904, 262]

Mais une fois ceci posé, Poincaré reste sceptique envers deux types de rigueur que nous allons caractériser : la rigueur de « l'analyste », qu'il juge mal adaptée à l'invention mathématique tout en lui reconnaissant une légitimité, et la « rigueur »du « logisticien », qu'il juge elle véritablement néfaste.

En 1902, Poincaré oppose deux types d'esprits mathématiques : «l'analyste » caractérisé par la rigueur de ses raisonnements et « le géomètre »caractérisé au contraire par son manque de rigueur et son appel à une intuition pensée au premier abord comme étant avant tout heuristique et ne donnant pas de certitude ${ }^{11}$ [Poincaré 1902]. Rappelons que Poincaré n'entend pas par « le géomètre » le mathématicien qui étudie la géométrie, ni par «l'analyste » celui qui étudie l'analyse, mais deux types distincts de méthodologie qui peuvent s'appliquer à toutes les branches des mathématiques, l'une étant plutôt attachée à la représentation géométrique, et l'autre à la rigueur analytique. Cette rigueur est caractérisée au premier abord comme voulant éliminer au maximum tout appel à l'intuition, mais comme nous le verrons par la suite, il y a bien selon Poincaré un type d'intuition qui joue un rôle dans la rigueur de l'analyste.

Pour préciser sa distinction, il compare « la conception géométrique de Riemann et la conception arithmétique de Weierstrass » en théorie des fonctions :

Weierstrass ramène tout à la considération des séries et à leurs transformations analytiques; pour mieux dire, il réduit l'Analyse à une sorte de prolongement de l'Arithmétique; on peut parcourir tous ses Livres sans y trouver une figure. Riemann, au contraire, appelle tout de suite la Géométrie à son secours, chacune de ses conceptions est une image que nul ne peut oublier dès qu'il en a compris le sens. [Poincaré 1902, 117]

Mais les travaux de Riemann manqueraient de rigueur, et cette rigueur n'aurait été obtenue par Weierstrass « qu'au prix de modifications profondes et de détours compliqués » qui en dénaturent le sens géométrique et masquent l'origine des idées. Cette opposition entre la démarche de Riemann et celle de Weierstrass est assez classique à son époque, notamment dans le sillage de Félix Klein [Klein 1898]. Néanmoins, Poincaré se démarque explicitement des conceptions de Félix Klein qui, selon lui, fait reposer le succès la méthode géométrique sur une intuition de type physique, alors que ce type d'intuition « ne peut nous donner la rigueur, ni même la certitude» qui sont bien pour Poincaré des caractéristiques essentielles du raisonnement mathématique. Il est ainsi indispensable de souligner que même si Poincaré part des points de référence de son époque, il leur donne un sens nouveau. Ainsi, chez Poincaré,

11. Nous pourrions donner comme exemple, non mentionné par Poincaré, le fait de compter les trous pour obtenir les nombres de Betti. 
le clivage n'oppose pas une arithmétique ou une analyse rigoureuse mais nonintuitive avec une géométrie moins rigoureuse mais plus intuitive et féconde. La distinction entre arithmétique et géométrie ne recoupe pas la distinction logique/sensible, car le raisonnement de type arithmétique pur n'est pas de la logique, et le raisonnement de type géométrique pur n'est pas empirique. Et pour cause, les mathématiques nécessitent un type de jugement qui ne soit ni empirique ni analytique, et qui repose donc sur une «intuition pure », point sur lequel Poincaré rejoint Kant. Toutefois, l'intuition chez Poincaré est très différente de chez Kant.

Pour clarifier le propos, il faut commencer par distinguer les intuitions de type empirique, qui fournissent des arguments heuristiques qui ne donnent pas de certitude, et que Poincaré appelle également « l'appel aux sens et à l'imagination » [Poincaré 1902]. Ceux-ci sont donc condamnables du point de vue de la rigueur. Mais de manière assumée, Poincaré revendique le fait qu'ils sont utiles, et donc que les normes de rigueur de l'analyste sont un peu trop strictes dans le cadre de la recherche mathématique. Nous avons ainsi vu que son Analysis Situs était bien à l'image de ce scepticisme. À ce propos, le point de vue de Jean Dieudonné dans [Dieudonné 1989] est intéressant pour nous car représentatif de celui d'un «analyste» au sens de Poincaré. Or, il porte un jugement très sévère sur la validité de ce mémoire, qu'il qualifie ainsi : « an unsuccessful attempt to give a genuine mathematical formulation to the intuitive ideas of Riemann and Betti ». À plusieurs reprises, Dieudonné se lamente des erreurs et des manques de rigueur qui le conduisent à ce jugement sévère. Bien sûr, il reconnaît la puissance et l'importance de ses idées, mais il n'y voit pas d'excuses pour un tel manque de précision. Ainsi, selon Dieudonné, dans l'Analysis Situs de 1895, « we should not look for precise definitions », et surtout «For many results, he simply gave no proof at all $»$ ou de temps en temps « an obscure and totally unconvincing argument ».

Une première lecture pourrait consister à dire que Poincaré reconnaît la nécessité de la rigueur, mais qu'il préfère laisser ce travail à d'autres. Et que le seul problème serait quand le manque de rigueur conduit à des erreurs mathématiques. Par exemple, Poincaré à partir de 1898 citera souvent comme véritable erreur commise par manque de rigueur l'ancienne croyance qu'une courbe continue admet nécessairement des tangentes en certains points ${ }^{12}$ [Poincaré 1898]. Selon lui, c'est ce contre-exemple qui motive l'arithmétisation de l'analyse, programme dont l'objectif est d'éliminer des raisonnements les appels à certaines intuitions.

Or, Poincaré affirme que même du point de vue de la rigueur la plus stricte, il faut l'usage d'un autre type d'intuition, qui est elle purement mathématique,

12. Nous pourrions cependant objecter à Poincaré qu'il n'y a pas ici d'erreur mathématique proprement dite, mais simplement une confusion entre deux notions distinctes de ce qu'est une courbe continue : celle définie à la manière de Weierstrass, et l'intuition originelle que nous en avions. De la même manière que la définition donnée par Poincaré des nombres de Betti ne correspondait pas à l'intention originelle. 
et qui serait même nécessaire pour obtenir la rigueur et la certitude. C'est donc bien une forme d'intuition totalement distincte de la précédente. L'exemple qu'il donne est «l'intuition du nombre pur » [Poincaré 1902], qui selon lui permet aux analystes «non seulement de démontrer, mais encore d'inventer ». La rigueur de l'analyste consisterait donc en réalité à réduire les mathématiques à l'intuition arithmétique pure. Ainsi, selon Poincaré, la validité du raisonnement par récurrence, qui permet de produire des connaissances arithmétiques, nous est donnée par l'intuition du nombre pur. Il n'y a donc pas à chercher à justifier ce raisonnement par des principes logiques. En affirmant cette position, principalement à partir de 1905 Poincaré s'oppose à ceux qu'il appelle les «logisticiens » et qui sont les logiciens comme Bertrand Russell ou Louis Couturat qui cherchent à réduire le nombre entier à une définition purement logique [Poincaré 1905].

Ainsi, Poincaré refuse la doctrine logisticienne comme étant celle qui essaie de chasser des mathématiques les intuitions pures, ce qui constitue déjà un dévoiement des objectifs de rigueur fixés par les analystes. Nous nous trouvons donc en présence de deux conceptions distinctes de la rigueur, chacune étant contestée par Poincaré : la conception des analystes, à laquelle il reproche de ne pas être adaptée à un travail de recherche (mais à laquelle il reconnaît le caractère rigoureux), et la conception des logisticiens qu'il juge véritablement erronée puisqu'elle élimine un type d'intuition dont les mathématiques ne peuvent pas se passer. Il suggère d'ailleurs que l'entreprise des logisticiens est vouée à l'échec même en suivant leurs propres critères. Poincaré s'amuse ainsi des cercles vicieux dans les définitions proposées des nombres entiers, bien qu'il précise que de toute façon, même si une définition logiquement satisfaisante finissait par être trouvée, cela ne changerait rien :

Vous donnez du nombre une définition subtile; puis une fois cette définition donnée, vous n'y pensez plus; parce qu'en réalité, ce n'est pas elle qui vous a appris ce qu'est un nombre, vous le saviez depuis longtemps. [Poincaré 1905, 821]

Ainsi, selon notre interprétation de Poincaré, vouloir définir les nombres entiers, c'est déjà renoncer à une certaine rigueur mathématique qui impose d'accepter cette notion comme fondamentale ${ }^{13}$. La conception logisticienne de la rigueur est donc disqualifiée. Mais ne peut-on pas aller plus loin, et chercher chez Poincaré une conception de la rigueur qui améliorerait celle des analystes?

13. Comme nous le verrons, il n'y a d'ailleurs pas que les nombres entiers qui doivent être admis pour Poincaré. 


\section{Vers une conception alternative de la rigueur mathématique}

En fait même en mettant de côté les considérations proprement heuristiques ou psychologiques qui doivent être disjointes de la rigueur elle-même, nous trouvons chez Poincaré des raisons précises conduisant à rejeter certaines normes trop strictes, au profit de l'usage assumé de certains types « d'intuitions ». Après les avoir présentées, il nous restera à proposer une interprétation liant ces raisons à ce que nous avons pu observer dans sa pratique des mathématiques.

Nous reprenons ici la thèse développée par Michael Detlefsen en 1992 [Detlefsen 1992]. Selon lui, Poincaré avance une conception de la « rigueur mathématique » différente de celle de la « rigueur logique ». Detlefsen distingue ainsi les inférences logiques (qui sont valides quel que soit le contexte) des inférences mathématiques (qui ne sont valides que dans un contexte mathématique précis). Il précise également :

It is important to distinguish the notion of a proposition's mathematically resting on another from that of a proposition logically resting on another. Propositions that belong to the logical basis of a proposition need not belong to its mathematical basis. [Detlefsen 1992, 367]

Pour expliquer cela, Detlefsen part d'un « principle of epistemic typification » qui sépare le domaine de nos connaissances en plusieurs types, dont le type mathématique et le type logique ${ }^{14}$. À cela il ajoute un «principle of epistemic conservation » qui dit que chaque domaine possède ses propres types d'inférences, et donc que pour le cas particulier des mathématiques, il faut utiliser des inférences de type mathématique. Poincaré combattrait donc la vision logicienne d'une preuve rigoureuse que Detlefsen caractérise ainsi :

a rigorous proof is one in which all substantive (i.e., topic-specific) information has been driven out of the inference and into the axioms, thence to be explicitly registered in the premises of the proofs in which it is used. [Detlefsen 1992, 352]

Au contraire, il défendrait une vision alternative de la rigueur selon laquelle :

rigor will be achieved not by the elimination of logical or informational gaps separating premises from conclusions (hence, the elimination of substantive inference) but, rather, by the elimination of gaps in our mathematical understanding (and, hence, the elimination of inferences in which the premises do

14. Il faudrait néanmoins faire attention au fait que pour Poincaré, le raisonnement logique se caractérise par ce qui n'apporte pas de nouvelle connaissance. 
not constitute a good mathematical reason for the conclusion). [Detlefsen 1992, 352]

Or, nous soutenons que cette compréhension de la marche d'un raisonnement mathématique ne peut se faire que par l'usage des intuitions. En effet, nous avons vu que selon Poincaré les mathématiques nécessitent un type de raisonnement qui donne à la fois certitude et fécondité, et sans lequel il n'est pas possible de produire de nouvelles mathématiques. Poincaré est en effet l'héritier d'une tradition kantienne selon laquelle la déduction logique, par définition, ne peut pas et ne doit pas produire d'information qui n'était pas déjà contenue dans les prémisses. Il faut donc expliquer comment peuvent se produire de nouvelles connaissances mathématiques. La logique se retrouve alors en position d'auxiliaire permettant certes d'organiser les théories mathématiques ou d'en contrôler certaines erreurs, mais perd son statut potentiel de producteur de connaissance mathématique. De plus, tout possible rôle fondationnel de la logique est écarté : en effet, l'idée de Poincaré est que certains concepts mathématiques doivent être admis, et que la véritable rigueur nécessite de savoir les reconnaître et ne pas chercher à les démontrer (ni à tenter d'en démontrer la non-contradiction) sous peine de cercle vicieux. Par exemple, nous avons vu que le principe de récurrence était pour Poincaré indémontrable à partir des seuls principes logiques.

Or, si Poincaré insiste sur des exemples tirés de l'arithmétique (comme le principe de récurrence) pour montrer l'impossibilité d'atteindre la rigueur prônée par les logisticiens, ce ne sont pourtant pas les seuls cas d'intuitions pures qu'il nous donne. Il serait ainsi réducteur de limiter son propos à l'arithmétique : selon lui en géométrie aussi (et même plus précisément en topologie), le mathématicien fait nécessairement usage d'intuitions pures qu'il ne peut pas éliminer de ses raisonnements. Comme nous l'avons déjà rappelé en première partie, il affirme ainsi en 1912 :

Et voici ce qui fait pour nous l'intérêt de cette Analysis Situs; c'est que c'est là qu'intervient vraiment l'intuition géométrique.

[Poincaré 1912, 484]

Et de plus, il ajoute que «nous avons tous en nous l'intuition du continu d'un nombre quelconque de dimensions $\gg$. Toutefois, il ne développera pas ces propos (il meurt la même année), mais selon notre interprétation c'est en fait l'usage de cette intuition géométrique pure qui distingue le « géomètre » de «l'analyste», et qui rend le raisonnement géométrique véritablement rigoureux. Certes, nous avons vu qu'en 1902, Poincaré pointait le manque de rigueur du géomètre, mais à cette époque il n'évoquait pas non plus d'intuition géométrique pure, comme s'il lui avait fallu quelques années pour réaliser la portée philosophique de ses travaux mathématiques en topologie. Selon nous, Poincaré a évolué sur ce point : il y a bien en réalité une rigueur proprement géométrique, distincte de la rigueur de l'analyste, et c'est celle à l'œuvre dans l'Analysis Situs. Le défaut de l'analyste serait ainsi de se limiter à la seule intuition du nombre pur, ce qui 
constitue bien un progrès par rapport à la méthode géométrique naïve, mais qui n'est pourtant pas suffisante pour développer les mathématiques. C'est pourquoi Poincaré souligne en 1908 :

les progrès de l'Arithmétique ont été plus lents que ceux de l'Algèbre et de l'Analyse, et il est aisé de comprendre pourquoi. Le sentiment de la continuité est un guide précieux qui fait défaut à l'arithméticien. [Poincaré 1908a, 934]

Mais la méthode géométrique pure (ou méthode topologique) se caractérise-t-elle uniquement par le fait de se reposer sur l'intuition pure du Continu, de la même manière que la méthode analytique se reposait sur l'intuition pure du nombre, ou bien y a-t-il une différence plus profonde entre les deux méthodologies? Nous avons vu que, pour Poincaré, la pratique des mathématiques, y compris rigoureuses, nécessite l'usage de certaines notions qui nous sont données directement par des intuitions pures qu'on ne peut pas remettre en doute. Poincaré distinguait bien ce type d'intuition de « l'appel aux sens et à l'imagination » [Poincaré 1902] auquel il reprochait son manque de rigueur, bien qu'il en soulignait l'utilité heuristique. Nous avons vu que cette distinction permettait de distinguer entre la rigueur de l'analyste (qui refuse tout appel aux intuitions sensibles) et la rigueur du logicien (qui refuse toute intuition).

Mais en fait, selon Poincaré, l'intuition ne sert pas uniquement à faire des généralisations heuristiques ou bien à garantir la certitude de ce que la logique ne peut pas fonder. Il y a également dans la pratique des mathématiques un usage d'un autre type d'intuition, qui correspond à la compréhension de la raison pour laquelle le mathématicien a fait tel ou tel choix. Il détaille ceci en 1908 [Poincaré 1908b], mais en avait déjà évoqué l'idée en 1902 [Poincaré 1902] : la pleine compréhension mathématique nécessite de prendre conscience du « je ne sais quoi qui fait l'unité de la démonstration». Selon Poincaré, c'est la faculté de percevoir les ressorts cachés d'une démonstration formelle qui fait que certaines personnes sont plus douées pour les mathématiques que d'autres. Mais comment cette faculté se manifeste-t-elle? Il parle de : « ce sentiment, cette intuition de l'ordre mathématique, qui nous fait deviner des harmonies et des relations cachées » nous permettant de comprendre le sens des démonstrations. Les 《relations cachées » correspondent à des liens entre des théories mathématiques a priori distinctes. C'est par la mise au jour de ces liens que les mathématiques progressent. Or, ce nouveau type d'intuition correspondrait à « un sentiment esthétique de la beauté mathématique, de l'harmonie des nombres et des formes, de l'élégance géométrique ». Notre hypothèse est que c'est l'usage de ce sentiment esthétique qui caractérise la véritable rigueur géométrique, ne pouvant pas être atteinte par l'analyste se limitant à la seule intuition du nombre pur.

En effet, du point de vue de l'analyste, tout pourrait idéalement se réduire à des définitions et des axiomes (qui correspondraient à ce que Poincaré appelle « intuitions pures ») à partir desquels on pourrait déduire logiquement 
les théorèmes. Mais ceci ne suffit pas à expliquer les pratiques mathématiques que nous avons observées en première partie : il faut comprendre pourquoi on a choisi telle définition plutôt qu'une autre. Or, dans l'exemple que nous avons observé, la bonne définition n'est pas venue au début d'une théorie, mais à la fin. Ce serait donc ici que nous aurions besoin de ce type d'intuition, qui permet de comprendre où on veut aller, et de «faire des choix» comme l'explique Poincaré. Pour illustrer ses propos, nous avons les exemples topologiques étudiés en première partie : nous avons vu que pour obtenir certaines propriétés des variétés, il a fallu les définir de différentes manières. Nous avons également vu que pour obtenir le théorème de dualité, il avait fallu sacrifier une certaine notion des nombres de Betti qui était pourtant, de l'aveu de Poincaré, celle portée par Riemann, Betti et lui-même au départ. Comment justifier qu'une notion soit sacrifiée au nom d'un théorème? Il existe des critères justifiant ces choix implicites qui constituent le véritable cœur d'une théorie mathématique, bien plus que les démonstrations qui ne sont généralement que le déroulement des propriétés que le mathématicien a sélectionnées après coup, ce qui peut donner un caractère artificiel à son travail.

Pour désigner ces critères, nous pourrions parler de la recherche de théorèmes « beaux » ou « élégants », puisque c'est Poincaré lui-même qui emploie cette terminologie. Mais encore faut-il donner un sens mathématique précis à ces termes. Dans l'exemple du théorème de dualité, on peut considérer l'apparition d'une symétrie comme un trait de beauté ou d'élégance. Certes ce sentiment esthétique peut paraître subjectif, mais ce qui importe avant tout c'est de comprendre quel est le résultat auquel voulait arriver le mathématicien à l'origine de la théorie considérée. Ainsi de manière générale, il est possible qu'on touche là à une limite du langage mathématique qui nécessiterait justement de s'en remettre à l'intuition du mathématicien. Mais quoi qu'il en soit, il reste possible pour le lecteur de chercher à retrouver les raisons qui ont motivé son choix. Et ceci est non seulement possible, mais même nécessaire pour parvenir à la bonne compréhension d'une théorie. Or, nous faisons l'hypothèse que seule l'utilisation d'un langage géométrique (par exemple les symétries) permet d'exhiber cette «harmonie cachée». Voici pourquoi la rigueur de l'analyste, quoi que valable, serait insuffisante pour prétendre rendre compte de ce qu'est la certitude mathématique.

Or, selon nous, c'est la topologie qui joue le rôle d'unification des mathématiques. En effet, cette conception des mathématiques semble avoir été profondément influencée par sa pratique de la topologie, à laquelle il est arrivé en explorant les bases de diverses théories mathématiques ${ }^{15}$, et qu'il a ensuite

15. Comme nous l'avions rappelé au début, il disait en 1901 : «Quant à moi, toutes les voies diverses où je m'étais engagé successivement me conduisaient à l'Analysis Situs. J'avais besoin des données de cette science pour poursuivre mes études sur les courbes définies par les équations différentielles et pour les étendre aux équations différentielles d'ordre supérieur et en particulier à celles du problème des trois corps. J'en avais besoin pour l'étude des fonctions non uniformes de 2 variables. J'en avais besoin pour l'étude des périodes des intégrales multiples et pour l'application de 
développée bien plus en se fiant à son sentiment esthétique qu'à des normes de rigueur strictes. Ainsi, la topologie fournit une nouvelle interprétation des autres disciplines mathématiques, leur donnant un sens nouveau permettant de les enrichir. Or, il faut bien préciser que dans cette perspective, la topologie ne serait pas une théorie servant à fonder les autres disciplines mathématiques. En effet, celles-ci sont également mobilisées pour faire progresser la topologie, par exemple la théorie des groupes pour étudier les variétés : elles ne peuvent donc pas être logiquement postérieures à la topologie. La topologie serait donc le cœur des mathématiques, mais pas son fondement.

\section{Conclusion}

En conclusion, nous affirmons que la conception «poincaréenne » de la véritable rigueur mathématique cumule plusieurs aspects. Si Poincaré n'a pas explicitement proposé cette interprétation, du moins nous estimons que celle-ci permet de donner une vision unifiée et cohérente de ses différentes remarques et pratiques. Tout d'abord, il s'agit de faire la place à l'usage d'intuitions pures permettant la production de nouvelles connaissances mathématiques. Mais pour aller plus loin, pour comprendre pour quelles raisons des systèmes mathématiques a priori distincts se rejoignent et permettent la démonstration de nouveaux théorèmes, il faut employer l'intuition topologique pure. Cette intuition topologique se caractérise par le fait qu'elle pose comme acquises les propriétés fondamentales de la continuité, et également qu'elle est capable de relier entre eux des domaines mathématiques a priori séparés en dévoilant des relations considérées comme « harmoniques ». Or, c'est précisément en cherchant les conditions pour que des objets satisfassent ces relations que le mathématicien pourra adopter les bonnes définitions des notions fondamentales. Et c'est donc bien la compréhension profonde des motivations esthétiques présidant au choix des définitions qui permet au lecteur de saisir le véritable sens de la démonstration : non pas des définitions aux théorèmes, mais l'inverse. Et c'est pour pouvoir exprimer mathématiquement ces motivations esthétiques que nous avons besoin de développer le langage topologique. C'est la raison profonde pour laquelle la topologie se retrouve être au cœur des mathématiques.

\section{Bibliographie}

Barrow-Greene, June [1997], Poincaré and the Three Body Problem, London : London Mathematical Society.

cette étude au développement de la fonction perturbatrice. Enfin j'entrevoyais dans l'Analysis Situs un moyen d'aborder un problème important de la théorie des groupes, la recherche des groupes discrets ou des groupes finis contenus dans un groupe continu donné » [Poincaré 1921, 101]. 
Darboux, Gaston [1913], Éloge historique d'Henri Poincaré, Paris : GauthierVillars.

Detlefsen, Michael [1992], Poincaré against the logicians, Synthese, 90(3), $349-378$.

Dieudonné, Jean [1989], A History of Algebraic and Differential Topology 1900-1960, Boston : Birkhäuser.

KLein, Félix [1898], Conférences sur les mathématiques, Paris : A. Hermann, trad. L. Laugel.

Poincaré, Henri [1895], Analysis Situs, Journal de l'École polytechnique, 1, $1-123$. 1-18.

[1898], L'œuvre mathématique de Weierstrass, Acta Mathematica, 22,

— [1899], Complément à l'Analysis Situs, Rend. Circ. Mat. Palermo, 13, $1-121$.

- [1902], Du rôle de l'intuition et de la logique en mathématiques, dans Comptes rendus du IIe Congrès international des mathématiciens, edité par E. Duporcq, Paris : Gauthier-Villars, 115-130.

- [1903], L'espace et ses trois dimensions, Revue de métaphysique et de morale, 11, 281-301.

— [1904], Rapport sur les travaux de M. Hilbert, Bulletin de la société physico-mathématique de Kasan, 14, 10-48.

- [1905], Les mathématiques et la logique, Revue de métaphysique et de morale, $13,815-835$.

- [1906], Les mathématiques et la logique, suite, Revue de métaphysique et de morale, 14, 294-317.

- [1908a], L'avenir des mathématiques, Revue générale des sciences pures et appliquées, 10, 930-939.

— [1908b], L'invention mathématique, L'Enseignement mathématique, 10, $445-459$.

- [1912], Pourquoi l'espace à trois dimensions, Revue de métaphysique et de morale, 20, 483-504.

_ [1921], Analyse des travaux scientifiques de Henri Poincaré faite par luimême, Acta Mathematica, 38, 3-135. 